\title{
Phytobiome as a Potential Factor in Nitrogen-Induced Susceptibility to the Rice Blast Disease
}

\author{
*Corresponding author \\ Tel: +82-53-810-3030 \\ Fax: +82-53-810-4769 \\ E-mail:jjeon@yu.ac.kr \\ ORCID \\ https://orcid.org/0000-0002-0617-4007
}

Received September 6, 2019

Revised September 19, 2019

Accepted September 20, 2019

\author{
Junhyun Jeon*(i) \\ Department of Biotechnology, College of Life and Applied Sciences, Yeungnam University, Gyeongsan \\ 38541 , Korea
}

\begin{abstract}
Roles of nutrients in controlling plant diseases have been documented for a long time. Among the nutrients having impact on susceptibility/resistance to crop diseases, nitrogen is one of the most important nutrients for plant growth and development. In rice plants, excess nitrogen via fertilization in agricultural systems is known to increase susceptibility to the rice blast disease. Mechanisms underlying such phenomenon, despite its implication in yield and sustainable agriculture, have not been fully elucidated yet. A few research efforts attempted to link nitrogen-induced susceptibility to concomitant changes in rice plant and rice blast fungus in response to excess nitrogen. However, recent studies focusing on phytobiome are offering new insights into effects of nitrogen on interaction between plants and pathogens. In this review, I will first briefly describe importance of nitrogen as a key nutrient for plants and what changes excess nitrogen can bring about in rice and the fungal pathogen. Next, I will highlight some of the recent phytobiome studies relevant to nitrogen utilization and immunity of plants. Finally, I propose the hypothesis that changes in phytobiome upon excessive nitrogen fertilization contribute to nitrogen-induced susceptibility, and discuss empirical evidences that are needed to support the hypothesis.
\end{abstract}

Keywords: Nitrogen-induced susceptibility, Phytobiome, Plant immunity, Rice blast disease

\section{Impact of Nitrogen on Susceptibility to Plant Diseases}

Crop diseases are one of the major limiting factors in agricultural production systems (Strange and Scott, 2005). Currently, control of diseases is heavily dependent on use of chemicals such as fungicides. Chemical-based disease control measures, though highly effective, pose serious threats to food and environmental safety, and raise concerns about development of resistance to agrochemicals among pathogen populations (Ishii, 2006). Breeding and deploying resistant crop varieties is

Research in Plant Disease

pISSN 1598-2262, elSSN 2233-9191

www.online-rpd.org an environment-friendly alternative, although their use is also limited by rapid emergence of pathogen strains that overcome host resistance in the field (Nelson et al., 2018).

Nutrients are important for growth and development of both plants and microorganisms associated with plants (Dordas, 2008; van Bruggen and Finckh, 2016). It has long been known that nutrients can influence outcome of interactions between plants and pathogens (Huber and Haneklaus, 2007). A number of studies have demonstrated the importance of proper nutrient management in attaining effective disease control and higher yield, simultaneously (Harrison and Shew, 2001; Heckman et al., 2003; Hoffland et al., 1999; Huber and Watson, 1974). Management of nutrients availability is required for full expression of genetic resistance, and is probably the most practical control measure for many plant diseases for which genetic resistance is not available (Dordas, 
2008). It should be, however, noted that there is no general rule regarding how the level of a particular nutrient can increase or decrease disease severity and incidence. High/low level of a nutrient, therefore, may increase disease severity in one crop species, but may have completely opposite effect on other crop species (Huber and Haneklaus, 2007).

Nitrogen $(\mathrm{N})$ is one of the most important nutrients for plant growth and development, and thus, nitrogen fertilization is routinely applied to increase crop yields (Huber and Watson, 1974). There is a volume of literatures about the effect of nitrogen on crop diseases (Celar, 2003; Harrison and Shew, 2001; Hoffland et al., 2000; Huber and Watson, 1974; Reid et al., 2001). Just as other nutrients, reports on the effect of nitrogen supply in disease development are inconsistent among different crop species, suggesting that effects of nitrogen on crop diseases are dependent on host-pathogen combination. It is frequently observed that high nitrogen is consistently associated with increase in susceptibility to biotrophic and hemi-biotrophic pathogens (Dordas, 2008). In case of rice, high nitrogen was shown to enhance susceptibility to the rice blast disease caused by Magnaporthe oryzae, and termed in a recent work as nitrogen-induced susceptibility (NIS) (Ballini et al., 2013; Huang et al., 2017). The molecular mechanisms of this phenomenon have not been clearly elucidated yet. Here, I summarize the recent molecular work on NIS and some of the phytobiome studies providing insights into nitrogen-use efficiency (NUE) and immune responses in plants. I argue that microbiome in plant might be a pivotal component in NIS and other nutrient-related modulation of plant immunity.

\section{Effects of High Nitrogen on Rice Plant and the Rice Blast Fungus}

Ballini et al. (2013) set up an experimental system, through which they could monitor NIS in rice without significantly altering plant development, and explored the relationship between NIS and genetic diversity of rice plants. They found that excess $\mathrm{N}$ supply does not significantly affect major $\mathrm{R}$ gene-mediated resistance, while it could modulate partial resistance of some cultivars. In plants supplemented with high $\mathrm{N}$, growth of the fungus seems to be faster than that in low $\mathrm{N}$, although penetration rate itself was not affected. The authors extended their observation to conclude that susceptibility to the wheat blast is affected by nitrogen supply as well, suggesting the generality of NIS.

In a follow-up study, they grew the plants in different $\mathrm{N}$ regimes (low and high $\mathrm{N}$ ) and compared transcriptomes of rice plants infected with the rice blast fungus by conducting dual RNA-sequencing (RNA-seq) experiment, in which transcripts of both host and pathogen are monitored in parallel, in an attempt to understand the molecular underpinnings of NIS (Huang et al., 2017). On the contrary to expectation, RNA-seq data revealed that some of the defense genes in rice are over-expressed by infection under high $\mathrm{N}$ regime. On the other hand, the fungus tends to express some effector genes and pathogenicity genes at higher level under high $\mathrm{N}$ regime than low $\mathrm{N}$ regime. From these results, the authors concluded that despite strong induction of plant defense responses, high $\mathrm{N}$ promotes fungal virulence enough to override such elevation of plant defenses, leading to increased number of lesions and changes in lesion types. During their analysis of RNA-seq data, it was found that most of genes involved in $\mathrm{N}$ metabolism showed little change between high and low $\mathrm{N}$ regime. However, expression of a glutamine synthetase in rice, OsGS1-2 increased at a higher level under high $\mathrm{N}$ than under low $\mathrm{N}$ regime. Importantly, they showed NIS is abolished in knockout mutant of OsGS1-2. This line of studies shed light on the molecular nature of NIS, and provided the basis for new hypotheses to be formulated and tested on this important phenomenon.

\section{Nitrogen Use and Phytobiome Composition}

Plants live in soil environments with diverse microbiota, and plant organs are intimately associated with a particular subset of these soil microbes (Berg and Smalla, 2009; Berg et al., 2016; Schlaeppi and Bulgarelli, 2015). In the study that are described above, Ballini et al. (2013) pointed out that NIS correlates with level of NUE, which is likely to be linked to regulation of nitrogen metabolisms involving OsGS1-2. Interestingly, a recent work on phytobiome (plant-associated microbiome) focused on the better NUE of indica varieties than that of japonica varieties in the field (Zhang et al., 2019), which are attributed, in part, to a natural variation in NRT1.1B encoding a nitrate transporter and sensor (Chen and Ma, 2015; Hu et al., 2015), and provided evidences for NRT1.1B being associated with the recruitment of a large proportion of indica-enriched bacteria. The authors of the work showed that indica-enriched bacteria had functions related to the nitrogen metabolism, and 


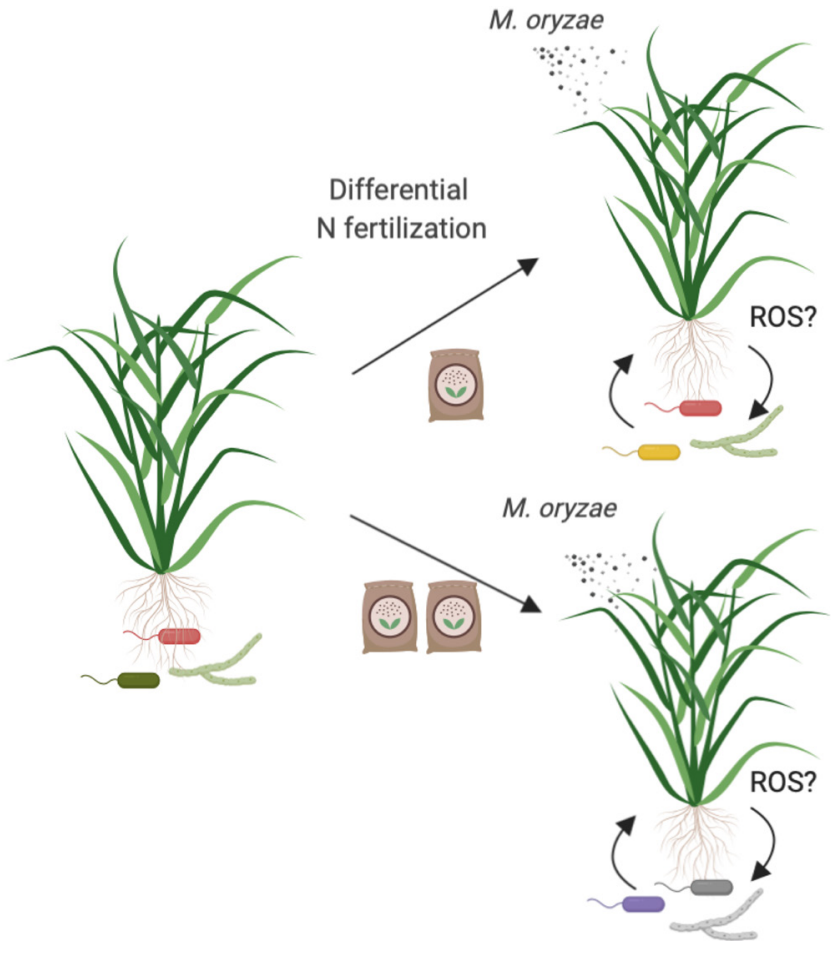

Fig. 1. Schematic diagram illustrating the effects of excessive nitrogen fertilization on susceptibility of rice plants to the rice blast disease. Shapes of different colors beneath the roots indicate different bacterial or fungal species/OTUs. Rectangles below arrows indicate nitrogen fertilization. ROS, reactive oxygen species; $M$. oryzae, Magnaporthe oryzae.

that synthetic community (SynCom) comprising of indicaenriched bacteria were, indeed, able to significantly promote plant growth when organic nitrogen was the sole nitrogen source. This study shows that rice plants can exert influence on shaping microbial communities to acquire nitrogen from the soil environment in order to optimize their growth.

Soil nitrogen is mainly present as nitrate, ammonium and organic nitrogen. Considering biochemical capacity of soil microbial communities in metabolizing such different forms of nitrogen, it is probably not too surprising that rhizosphere communities can account for, not all but significant part of, NUE variation among plants. An important question yet to be answered would be what physiological factors (such as root exudates) of plants govern its interactions with microbes and how these factors guide rhizosphere microbiome assembly (Sasse et al., 2018).

It has been well established that nutrient content of soil is able to change composition and structure of microbial communities (Fierer, 2017), which in turn have the potential to alter physiology and immunity of the plants. This fact, together with ability of plants to recruit particular subset of soil microbes for its own growth and development, strongly indicates the presence of positive or negative feedback among plants, microbes, and nutrient contents of soils. From this perspective, it is critical for better management of agricultural production systems to understand what changes nutrient content of soil can cause in microbial communities of soil, and in turn, what impact such changes in microbial communities have on plants.

\section{Nitrogen Supplies and Plant Immunity}

Plants have to optimize growth and defense using nutrients that are available from their environments. Failure to strike a balance between the two can lead often to devastating outcomes such as stunting or disease (Karasov et al., 2017). However, both excess as well as shortage of nutrients are problems for plants to adequately cope with. At least in Arabidopsis thaliana, shortage of $\mathrm{N}$ has been shown to result in over-production of reactive oxygen species (ROS) that is correlated with up-regulation of a gene encoding nitrate transporter, NRT2.1 (Bellegarde et al., 2019). N starvation, thus promotes lateral root growth and active transport of $\mathrm{N}$ into the roots. On the other hand, excess $\mathrm{N}$ triggers a histonemodifying enzyme of $A$. thaliana, which, in turn, is required for activating a suite of ROS detoxification genes, maintaining ROS homeostasis and preventing mis-regulation of NRT2.1 (Bellegarde et al., 2019).

Cellular ROS level is intimately related to regulation of both development and defense in plant. The fact that rice nitrate transporter and sensor, NRT1.1B is one of the primary factors in shaping root microbiome suggests that excess $\mathrm{N}$ through fertilization may impact root microbiome through changes in plant immunity, which are possibly mediated by ROS. Furthermore, such changes in microbial communities may have feedback effect on immunity in their host plant. Therefore, it can be hypothesized that such reciprocal changes under excess $\mathrm{N}$ condition might be a key component in NIS.

\section{What Empirical Evidences Are Needed to Rstab- lish Link between Nitrogen-Induced Susceptibil- ity and Microbiome?}

In order to investigate link between NIS and phytobiome, 
it is necessary to set up an experimental condition, in which effect of excess $\mathrm{N}$, provided in the form of fertilizer containing ammonium and nitrate, can be monitored without having pronounced effect on plant growth and physiology. This experimental setting should not change physico-chemical properties of soil to large degree. If application of $\mathrm{N}$ fertilizer leads to dramatic changes in $\mathrm{pH}$ for example, it would have confounding effects on both plants and soil microbial communities.

Once this system is set up, then one has to check whether soil and/or endophytic microbial communities change and then what are those changes. This can be done with relative ease through next-generation sequencing-based profiling of microbial communities. There might be a key taxon or taxa that are correlated with NIS, and they would be good candidate microbes that deserve further attention. Translation of correlation into causation requires two approaches: culture-dependent and culture-independent approaches. In culture-dependent approach, isolated microbes that are formulated into synthetic communities are used. In cultureindependent approach, microbial fraction of soils as a whole is used without isolation of individual microbes. One should test if either synthetic communities or microbial fraction can recapitulate NIS (at least partially) in rice plants without N fertilization, and should examine at the molecular level changes in plants that treatment of plants with NIS-associated microbes can cause. Such line of data would establish contribution of phytobiomes to NIS in rice plants.

\section{Conclusion}

We now start to appreciate that there exist complex relationships among phytobiome, plant immunity, and soil environment including nutrient content. Here I have discussed the possibility that phytobiome is a contributing factor to NIS in rice plants. Interaction among plants, nutrient contents, and phytobiome is a dynamic process, likely dependent on positive and/or negative feedbacks from one another. Key nutrients such as $\mathrm{N}$ or phosphate $(\mathrm{P})$ are limiting factor to both plants and micro-organisms in soil. Level of such key nutrients in soil is highly likely to impact microbial community composition and structures. Current agricultural systems rely on heavy use of fertilizers to increase crop yields. Such practices affect the level of nutrients available for plant and microbial communities harboring pathogens, which can af- fect the disease severity. From this perspective, understanding changes and identifying roles of phytobiome in response to fertilization are critical for devising cultural control for plant diseases in environment-friendly and sustainable manner via microbiome-informed nutrient manipulation.

\section{Conflicts of Interest}

No potential conflict of interest relevant to this article was reported.

\section{Acknowledgements}

This work was supported by a grant (PJ013178) from the Rural Development Administration, Republic of Korea.

\section{References}

Ballini, E., Nguyen, T. T. T. and Morel, J.-B. 2013. Diversity and genetics of nitrogen-induced susceptibility to the blast fungus in rice and wheat. Rice (NY) 6: 32.

Bellegarde, F., Maghiaoui, A., Boucherez, J., Krouk, G., Lejay, L., Bach, L. et al. 2019. The chromatin factor HNI9 and ELONGATED HYPOCOTYL5 maintain ROS homeostasis under high nitrogen provision. Plant Physiol. 180: 582-592.

Berg, G., Rybakova, D., Grube, M. and Koberl, M. 2016. The plant microbiome explored: implications for experimental botany. J. Exp. Bot. 67: 995-1002.

Berg, G. and Smalla, K. 2009. Plant species and soil type cooperatively shape the structure and function of microbial communities in the rhizosphere. FEMS Microbiol. Ecol. 68: 1-13.

Celar, F. 2003. Competition for ammonium and nitrate forms of nitrogen between some phytopathogenic and antagonistic soil fungi. Biol. Control 28: 19-24.

Chen, Z. C. and Ma, J. F. 2015. Improving nitrogen use efficiency in rice through enhancing root nitrate uptake mediated by a nitrate transporter, NRT1.1B. J. Genet. Genomics 42: 463-465.

Dordas, C. 2008. Role of nutrients in controlling plant diseases in sustainable agriculture: a review. Agron. Sustain. Dev. 28: 33-46.

Fierer, N. 2017. Embracing the unknown: disentangling the complexities of the soil microbiome. Nat. Rev. Microbiol. 15: 579-590.

Harrison, U. J. and Shew, H. D. 2001. Effects of soil pH and nitrogen fertility on the population dynamics of Thielaviopsis basicola. Plant Soil 228: 147-155.

Heckman, J. R., Clarke, B. B. and Murphy, J. A. 2003. Optimizing manganese fertilization for the suppression of take-all patch disease on creeping bentgrass. Crop Sci. 43: 1395-1398.

Hoffland, E., Jeger, M. J. and van Beusichem, M. L. 2000. Effect of nitrogen supply rate on disease resistance in tomato depends on 
the pathogen. Plant Soil 218: 239-247.

Hoffland, E., van Beusichem, M. L. and Jeger, M. J. 1999. Nitrogen availability and susceptibility of tomato leaves to Botrytis cinerea. Plant Soil 210: 263-272.

Hu, B., Wang, W., Ou, S., Tang, J., Li, H., Che, R. et al. 2015. Variation in NRT1.1B contributes to nitrate-use divergence between rice subspecies. Nat. Genet. 47: 834-838.

Huang, H., Nguyen Thi Thu, T., He, X., Gravot, A., Bernillon, S., Ballini, E. et al. 2017. Increase of fungal pathogenicity and role of plant glutamine in nitrogen-induced susceptibility (NIS) to rice blast. Front. Plant Sci. 8: 265.

Huber, D. M. and Haneklaus, S. 2007. Managing nutrition to control plant disease. Landbauforsch. Volk. 57: 313-322.

Huber, D. M. and Watson, R. D. 1974. Nitrogen form and plant disease. Annu. Rev. Phytopathol. 12: 139-165.

Ishii, H. 2006. Impact of fungicide resistance in plant pathogens on crop disease control and agricultural environment. Jarq-Jpn. Agric. Res. Q. 40: 205-211.

Karasov, T. L., Chae, E., Herman, J. J. and Bergelson, J. 2017. Mechanisms to mitigate the trade-off between growth and defense.
Plant Cell 29: 666-680.

Nelson, R., Wiesner-Hanks, T., Wisser, R. and Balint-Kurti, P. 2018. Navigating complexity to breed disease-resistant crops. Nat. Rev. Genet. 19: 21-33.

Reid, L. M., Zhu, X. and Ma, B. L. 2001. Crop rotation and nitrogen effects on maize susceptibility to gibberella (Fusarium graminearum) ear rot. Plant Soil 237: 1-14.

Sasse, J., Martinoia, E. and Northen, T. 2018. Feed your friends: do plant exudates shape the root microbiome? Trends Plant Sci. 23: 25-41.

Schlaeppi, K. and Bulgarelli, D. 2015. The plant microbiome at work. Mol. Plant-Microbe Interact. 28: 212-217.

Strange, R. N. and Scott, P. R. 2005. Plant disease: a threat to global food security. Annu. Rev. Phytopathol. 43: 83-116.

van Bruggen, A. H. C. and Finckh, M. R. 2016. Plant diseases and management approaches in organic farming systems. Annu. Rev. Phytopathol. 54: 25-54.

Zhang, J., Liu, Y.-X., Zhang, N., Hu, B., Jin, T., Xu, H. et al. 2019. NRT1.1B is associated with root microbiota composition and nitrogen use in field-grown rice. Nat. Biotechnol. 37: 676-684. 